\title{
A Case with Sneddon Syndrome Combining with Preeclampsia and Intrahepatic Cholestasis of Pregnancy
}

\author{
Ece Bahceci ${ }^{1(\mathbb{D})}$, Murat Hosgoren ${ }^{1(\mathbb{I D})}$, Keziban Dogan $^{1(\mathbb{I D})}$ Emine Ozturk $^{1(\mathbb{I D})}$ \\ ${ }^{1}$ Obstetrics and Gynecology Clinic, University of Health Sciences, Dr. Sadi Konuk Education and Research Hospital, Istanbul, \\ Turkey
}

Copyright@ Author(s) - Available online at https://dergipark.org.tr/en/pub/mbsjohs

Content of this journal is licensed under a Creative Commons Attribution-NonCommercial 4.0 International

License,

Received:16 October 2020, Accepted: 24 March 2021, Published online: 30 April 2021

(C) Ordu University Institute of Health Sciences, Turkey, 2021

\begin{abstract}
Sneddon's syndrome (SS) is a rarely seen thrombotic vasculopathy characterized by the combination of ischemic cerebrovascular disease with livedo racemose (LR) and mainly affects young women in the third decade of life. We present a case of an 32 year old women diagnosed with SS 17 years ago, admitted to our emergency department when she was 37 weeks pregnant, and diagnosed as having superimposed severe preeclampsia and intrahepatic cholestasis of pregnancy, who had an emergency cesarean section. There are very few case reports presenting the relationship between pregnancy and SS and a possible relationship between this syndrome and preeclampsia or cholestasis has not yet been shown. We think that our case presentation may become important to contribute literature.
\end{abstract}

Keywords: Sneddon Syndrome, Pre-Eclampsia, Cholestasis

Suggested Citation: Bahceci E, Hosgoren M., Dogan K, Ozturk E. A case with sneddon syndrome combining with preeclampsia and intrahepatic cholestasis of pregnancy. Mid Blac Sea Journal of Health Sci, 2021; 7(1):143-146

Address for correspondence/reprints:

Ece Bahceci

Telephone number: +90 (537) 4085754

E-mail: ecebahceci@yahoo.com 


\section{Introduction}

Sneddon's syndrome (SS) is a rare noninflammatory thrombotic vasculopathy of small and medium-sized arteries characterized by the combination of ischemic cerebrovascular disease with livedo racemose (LR) defined as net-like, patchy, violaceous, skin discoloration (1). It has been estimated that the incidence of SS is 4 per 1 million per year and mainly affects young women with a mean onset in the third decade of life, between the ages of 20 and 42 years (2). SS is an episodic or chronic, slowly progressive syndrome that was first described in 6 patients who had several common symptoms such as lacunar, subcortical, ischemic cerebral infarcts and widespread livedo eruption (3). LR may progress over the years and the trunk and/or buttocks are involved in nearly all patients. Recurrent cerebrovascular events are mostly secondary to ischemia including transient ischemic attacks and cerebral infarct (4). On the other hand; headache, cerebral hemorrhage, seizures, vertigo, cognitive and psychiatric disturbances, can be seen as neurological symptoms (5). Approximately $80 \%$ of patients with SS have an antiphospholipid antibody marker and other manifestations of primary antiphospholipid syndrome (1). It can be diagnosed by general LR with typical histopathological findings on skin biopsy and focal neurological deficits $(1,2)$. The pathogenesis of SS is associated with hypercoagulability and intrinsic small-vessel vasculopathy. The optimal therapy of SS remains an unresolved problem and long-term anticoagulation is recommended for cerebral ischemic events. But there is controversy about the treatment of SS with immunomodulatory agents (4). The relation of pregnancy and SS has been reported rarely and to our knowledge, there is no data to be found in the literature about the relationship and coexistence between SS and pregnancy complicated with preeclampsia and cholestasis. We aimed to contribute to the literature by presenting a rarely seen case of a pregnant woman with SS, complicated by preeclampsia and cholestasis.

\section{Case}

We report the case of a nulliparous 32-year-old pregnant woman who has SS since she was 15 . She experienced one ischemic attack at the time of her diagnosis. She was admitted to the emergency room of the obstetrics and gynecology department at her 37 weeks of pregnancy due to increased blood pressure and lack of variability in her nonstress test (NST). General examination showed erythematous macular eruption on her skin involving the abdominal region. Her symptoms were headache, palpitation, itching, dryness in her mouth, swelling in her feet, increasing pain in her hand, wrist, and knee. According to her last menstrual period, she had a pregnancy of 37 weeks and 1 day. In the fetal examination, a 37-week fetus with the head presentation was observed, her amniotic fluid index and fetal doppler findings were normal. There was no fetal demise detected. At the time of her admission to the hospital, her blood pressure was $170 / 100 \mathrm{mmHg}$, platelet count was $153000 / \mathrm{microL}$, liver enzymes were elevated (AST:70 U/L ALT:63 IU/L LDH:580 U/L). The serum total bile acid concentration was 72 micromol/L, bilirubin levels were normal. In her urine sample $(++)$ albumin was seen. The antiphospholipid antibodies were negative. Her electrocardiogram was normal. In echocardiography, ejection fraction was in the normal range, minimal aortic failure was noted. She had a history of chronic hypertension presumably due to the SS. The medication she was using; alpha-methyl-dopa $250 \mathrm{mg}$ tb $3 \times 2$ per oral (P.O)., methylprednisolone $2 \mathrm{mg}$ tb $2 \times 1$ P.O., azathioprine $50 \mathrm{mg}$ tb $2 \times 1$ P.O, ursodeoxycholic acid $250 \mathrm{mg}$ tb $2 \times 2$ P.O., enoxaparin sodium $40 \mathrm{mg} 1 \times 1$ subcutaneously (S.C.). In her previous visits, her blood pressure was in normal range with medical therapy Due to known chronic hypertension, headache, and high levels of protein in her urine; superimposed preeclampsia was diagnosed. Additionally, her liver enzymes and bile acid level were elevated. Cholestasis of pregnancy was added to our diagnosis. Due to our findings, pregnancy termination was planned. A consultation was made to her specialist in the department of rheumatology. Due to the risks of thromboembolism and stroke during the stages of normal vaginal labor, the rheumatologist recommended labor with cesarean section. Her blood pressure remained above $160 / 110 \mathrm{mmHg}$. The patient remained suffering from severe headaches. The nonstress test showed decreased variability. Magnesium sulfate protocol was initiated immediately, and the pregnancy was terminated with cesarean section. After the operation magnesium sulfate infusion was continued for 24 hours. The patient was re-evaluated by the rheumatology specialist postoperatively. The post-operative second day her blood pressure increased to $180 / 100 \mathrm{mmHg}$. We manage to control the blood pressure after giving $5 \mathrm{mg}$ of hydralazine. Nifedipine $30 \mathrm{mg}$ P.O. and Enoxaparin sodium $60 \mathrm{mg}$ S.C. was added to her routine treatment. The patient was observed in the hospital until the postoperative fourth day. She was discharged after her blood pressure remained regulated for 48 hours. During the patient's follow-up until postpartum 6th month, we did not observe any thromboembolic events. 
Although we had informed consent from the patient to make a case report, we cannot make a photo presentation because the patient did not allow us to take photographs of the skin lesions due to her religious beliefs.

\section{Discussion}

SS is a rare disease of unknown etiology with dermatological manifestation and recurrent strokes. It can be classified as, antiphospholipid syndrome associated, systemic lupus erythematosus related or idiopathic if there is no causative factor identified (4). In our case, it was classified as idiopathic because of no relation to SLE or antiphospholipid syndrome. Besides cutaneous and neurologic symptoms, systemic hypertension, valvular and ischemic heart disease, renal disease, retinopathy, Raynaud phenomenon, and fetal loss are also commonly seen in SS $(1,4)$. In our patient, there were no signs of cardiac, renal, or ophthalmic disease but she had chronic hypertension and no abortion history. The optimal management of patients with SS is yet an unresolved problem, there have been no prospective controlled studies to guide therapy because of the rare incidence of the disease. The main goal is to prevent further cerebrovascular events and to reduce skin symptoms. Antiplatelet therapy and anticoagulation both decrease the risk of secondary ischemic events. Recent research suggests that the prognosis of stroke recurrence might be better compared to the historical data due to the increasing use of antiplatelet/antithrombotic agents for secondary stroke prophylaxis (1). Also, our patient had one cerebral ischemic attack, no history of recurrent ischemic attacks and cerebral infarct due to the beneficial effect of anticoagulant therapy. Although it has been suggested that anti-inflammatory or immunosuppressive therapies are beneficial, because of the presence of an inflammatory phase in the early stage of SS, it has been shown that these therapies such as corticosteroids and azathioprine are generally not beneficial (1). But our patient was receiving corticosteroids and azathioprine and had used it throughout her pregnancy. When it comes to rare diseases; we believe that the relevant studies should be followed closely by the related branches and the previous experiences in the treatment should be taken into consideration. Since SS is seen frequently in women and in their fertile period; pregnancy, complications related to pregnancy and their management become important. In addition, the case reports presenting the relationship and prognosis of pregnancy and SS is very limited (6- 8). Dikova et al. (6) claimed an increase in abortion, similar to that
Baleva et al claimed an increase in recurrent abortion. In our patient, she did not experience any risk of abortion and it was her first pregnancy. She had regular follow-up throughout her pregnancy and underwent antihypertensive treatment because of a history of chronic hypertension. Her blood pressure levels were within normal limits until her emergency admission. Dietl et al. (8) presented a case of 31-yearold pregnant women with SS in 1991. She developed episodes of cerebral ischemia with multiple neurological deficits in the 24th week of pregnancy. The symptoms were associated with the dermatological signs of LR. In the 36th week of pregnancy, delivery was made by cesarean section and it resulted in a major improvement of the neurological signs and symptoms. The immunosuppressive therapy with cortisone and cyclophosphamide was planned but due to the persistent improvement in the patient's condition, it was not instituted. Our patient was 32 years old, diagnosed as SS at the age of 15 , had a cerebral ischemic attack without any neurological disability that responded to anticoagulant therapy. She had been given enoxaparin sodium $40 \mathrm{mg}$ 1x1 subcutaneously during pregnancy. When she was admitted to our emergency room, she had a 37-week pregnancy. She was diagnosed with high blood pressure, proteinuria, headache, edema in the hands and feet, pruritus due to bile acid elevation. She was diagnosed with superimposed severe preeclampsia and cholestasis, and the pregnancy was terminated with a cesarean section. On postpartum day four, blood pressure was under control and she was discharged. The limitation of our case report is the lack of photograph presentation. Nevertheless, to our knowledge, this is the first report presenting a pregnant woman with SS complicating with superimposed severe preeclampsia and cholestasis. Therefore, we think that it will contribute to the literature data. Despite insufficient data, it is known that pregnancy with SS is a high-risk pregnancy and the risk of abortion increases. Due to increased coagulability and vasculopathy in small vessels, the risk of preeclampsia and cholestasis may be increased as seen in our case. Our patient had been receiving anticoagulant treatment since diagnosis and she had no recurrent ischemic attacks. As anticoagulant therapy decreases cerebral ischemic attacks by reducing thrombosis it can also decrease the risk of abortion and preeclampsia. However, more study data is needed in this subject. 


\section{Conclusion}

We believe that pregnancy with SS is a high-risk condition and it should be followed with caution by experienced clinicians. Anticoagulant therapy can be considered during pregnancy until delivery as it may be helpful to decrease complications. Pre-eclampsia and cholestasis can increase mortality and morbidity therefore during these pregnancies suspicion and cautiousness are necessary.

Ethics Committee Approval: Approval was received for this study from the patient.

Peer-review: Externally peer-reviewed.

\section{Author Contributions:}

Concept: K.D, E.O, E.B. Design: E.B, M.H, Literature Search: K.D, E.O, E. B. Data Collection and Processing: K.D, E.O, E. B. Analysis and/or Interpretation: E. B, M.H, Writing: E.B, M.H,

Conflict of Interest: No conflict of interest was declared by the authors.

Financial Disclosure: The authors declared that this study hasn't received no financial support.

\section{References}

1 Samanta D, Cobb S, Arya K. Sneddon Syndrome: A Comprehensive Overview J Stroke Cerebrovasc Dis.2019; 8:2098-2108

2 Zelger B,Sepp N, Stockhammer G, et al. Fritsch PO. Sneddon's Syndrome:A Longterm Follow-up of 21 Patients Arch Dermatol. 1993;129:437-447.

3 Sneddon IB. Cerebrovascular lesions and livedo reticularis. Br J Dermatol. 1965;77:180-5.

$4 \mathrm{Wu} \mathrm{S}, \mathrm{Xu} \mathrm{Z}$, Liang H. Sneddon's syndrome: a comprehensive review of the literature Orphanet J Rare Dis. 2014;9:215.

5 Tourbah A, Piette JC,Iba-Zizen MT, Lyon-Caen $\mathrm{O}$, Godeau P,Francès C. The Natural Course of Cerebral Lesions in Sneddon Syndrome Arch Neurol 1997;54:53-60.

6 Dikova Ch, Kolarov G, Baleva M, Nikolov K. Spontaneous abortions in Sneddon's syndrome Akush Ginekol.2000;39:24-5.

7 Baleva M, Boyanovsky B, Nikolov K, Kolarov Z, Nikolova M. High levels of $\operatorname{IgA}$ anticardiolipin antibodies in patients with systemic lupus erythematosus, HenochSchoenlein purpura, Sneddon's syndrome and recurrent pregnancy loss. Thromb Haemost.1999;82:1774-5.

8 Dietl J, Stroppel G, Poremba M, Lubach D. Sneddon's syndrome and pregnancy; a case report. Eur J Obstet Gynecol Reprod Biol.1991 10;39:219-21 